\title{
Placental Insufficiency and Fetal Growth Restriction
}

\author{
Krishna Usha $\cdot$ Bhalerao Sarita
}

Received: 17 September 2011 / Accepted: 15 October 2011/Published online: 17 November 2011

(C) Federation of Obstetric \& Gynecological Societies of India 2011

\begin{abstract}
Objectives Fetal growth restriction is defined as a pathologic decrease in the rate of fetal growth. The most frequent etiology for late onset fetal growth restriction is uteroplacental dysfunction which is due to inadequate supply of nutrients and oxygen to support normal aerobic growth of the fetus. However, for symmetrical IUGR, fetal chromosomal anomalies, structural anomalies and fetal infections should be carefully excluded. Consequent to the uteroplacental vascular maladaptation of endovascular trophoblastic invasion, there is increased vascular resistance and decreased blood flow to the placenta in the choriodecidual compartment.

Conclusions This under perfusion of the placenta causes villous damage; that is, total tertiary villous capillary bed is reduced leading to increased placental resistance. These changes can be diagnosed by Doppler and characteristic changes are seen in the uterine, umbilical, middle cerebral arteries and ductus venosus vessels. In severe cases, delivery of the fetus with optimum intrapartum surveillance, or caesarean section, is essential.
\end{abstract}

Krishna U., Consultant Obstetrician and Gynaecologist AjitVilla, Laburnum Road, Gamdevi., Mumbai 400007, India

Bhalerao S., Consultant Obstetrician and Gynaecologist Bhatia, Saifee, St. Elizabeth's and Wadia Hospital, Mumbai, India e-mail: saritabhalerao@hotmail.com

Krishna U. ( $\varangle$ ), Consultant Obstetrician and Gynaecologist Breach Candy, Bhatia and St. Elizabeth's Hospitals, Mumbai, India e-mail: drushark@sify.com
Keywords IUGR · Placental insufficiency · Colour doppler $\cdot$ Low birth weight

\section{Introduction}

Fetal weight is determined by the genetic growth potential, the health of the fetus, the capacity of the mother to supply adequate quality and quantities of substrates required for growth and the ability of the placenta to transport these nutritional substrates to the fetus. The majority of conditions affecting fetal growth are placental or fetal in origin.

The most common placental conditions are alterations in the uteroplacental and fetal-placental circulations. In the majority of these cases, there is diminished maternal uteroplacental blood flow, caused by insufficient or incomplete trophoblastic invasion of the spiral arteries in the placental bed.

\section{Causes of Fetal Growth Restriction}

The causes can be broadly classified as fetal, maternal and placental factors.

Fetal causes include chromosomal abnormalities, multiple pregnancies, fetal structural anomalies and fetal infections.

Maternal factors include nutritional deficiencies, especially of vitamin C and E. Maternal infections, especially chronic infections like tuberculosis and malaria can cause fetal growth restriction (FGR). 
Medical problems in the mother like hypertension, anemia, diabetes mellitus, chronic lung diseases and heart disease can cause FGR.

Antiphospholipid antibody (APA) syndrome is associated with first trimester miscarriage, intrauterine death, preeclampsia, fetal growth restriction. Antigen-antibody binding and complement activation increases thrombogenic potential and leads to widespread endothelial damage. Vasculopathy of the spiral arteries leads to placental infarcts and thrombosis.

It has been suggested that oxidative stress is one of the causes of FGR. Karowicz-Bilinska et al. [1] found elevated values of indices of oxidative stress in the serum of pregnant women with IUGR.

\section{Diagnosis of FGR}

In order to diagnose FGR it is essential to estimate gestational age accurately. Although this is usually calculated from the last menstrual period, when known with certainty, the reliability of this estimate is low as timing of ovulation is variable. A first trimester ultrasound can date the pregnancy more reliably.

In both developed and developing countries and for all racial and ethnic groups, there is a positive relationship between maternal total weight gain and fetal birth weight. Inadequate weight gain(less than $4.3 \mathrm{~kg}$ ) in early pregnancy, earlier than 24 weeks is an independent predictor of low birth weight or SGA.

\section{Serial Fundal Height Estimation}

Serial fundal height estimation is a simple technique for assessing fetal growth. The measurement begins from the fundus till the symphysis pubis with a non-elastic tape with its centimeter side facing-down to avoid manipulation.

\section{Biochemical Markers}

Coyle and Brown in 1963 reported significantly lower urinary estriol levels in pregnancies with small babies. The development of radioimmunoassay promoted a shift from urinary to blood estriol. Unfortunately significant day to day variability in the urinary excretion level and diurnal variation in plasma concentration made interpretation difficult. Human placental lactogen was first promoted in the late 1960s as a marker of placental function. Studies of normal and abnormal pregnancies let to the concept of the fetal danger zone which classified plasma hPL concentrations below $4 \mu \mathrm{g} / \mathrm{ml}$ after 30 weeks gestation as abnormally low and indicative of a pregnancy at grave risk.
However, as a significant number of fetal deaths could occur in association with normal concentrations of hPL, the assay did not find a wide application [2].

\section{Ultrasonographic Biometry}

Ultrasound measurements of the biparietal diameter, head circumference, abdominal circumference and femur length are recorded and matched with the 50th percentile of the corresponding parameter in readymade population charts. Measurements below the 10th percentile are highly suspicious of FGR and measurements below 3rd percentile are unequivocal evidence of FGR. Growth in the abdominal circumference of less than $1 \mathrm{~cm}$ over 14 days is also indicative of FGR.

Ponderal Index

Estimated fetal weight [3] less than the 10th percentile. Based on the Ponderal index two types of FGR are described:

1. Symmetric FGR These infants have a normal Ponderal index in which weight and length are growth restricted and infants have a small head circumference. Early onset growth restriction is presumed

2. Asymmetric FGR These babies have a low Ponderal index in which weight is restricted more than length. Here there is late onset growth restriction

Amniotic fluid is derived from fetal urine and from respiratory tract. In FGR, shunting of blood from the splanchnic circulation results in reduced renal blood flow, reduced glomerular filtration rate and hence less liquor. The amniotic fluid index is measured by adding the vertical depth of cord free amniotic fluid pools in each of the four uterine quadrants. A combined depth of $5 \mathrm{~cm}$ or more is normal. Similarly a single vertical pocket of amniotic fluid of more than $2 \mathrm{~cm}$ is considered as normal.

Another clue to the presence of FGR is presence of placental calcium deposits, indicating placental senescence. A finding of a grade 3 placenta before 36 weeks is corroborative evidence of FGR [3].

\section{Role of Doppler}

\section{Uterine Artery Doppler}

The majority of blood flow to the uterus is supplied by the uterine arteries. Throughout gestation, uterine blood flow increases 10-12 fold due to trophoblastic invasion of the spiral arteries within the myometrium and decidua, and $50 \%$ increase in maternal blood volume. The shape of the 
uterine artery Doppler waveform is unique and changes as gestation advances. In early pregnancy, the uterine circulation is characterized by high vascular impedance and low flow, giving a waveform with persistent end-diastolic velocity and continuous forward blood flow throughout diastole. As the trophoblastic invasion and spiral artery modification proceed, placental perfusion increases and the uteroplacental circulation becomes a high-flow, low-resistance system giving a waveform with greater end-diastolic flow.

When the normal trophoblastic invasion and modification of spiral arteries is interrupted, there is increased impedance to flow within the uterine arteries and decreased placental perfusion. These pathological processes are key features common to the development of pre-eclampsia and IUGR.

A prospective trial by Zimmermann et al. [4] evaluated the utility of uterine artery Doppler performed between 21 and 24 weeks in the prediction of subsequent development of pre-eclampsia and IUGR. They identified 175 women at high risk for developing hypertensive disorders of pregnancy or IUGR, and 172 low-risk pregnancies. Persistent notching or elevated RI in the uterine arteries or an elevated RI in the uteroplacental arteries were defined as pathological Doppler signs.

Based on the currently available data, there is insufficient evidence to recommend uterine artery Doppler as a general screening modality for all pregnancies. When performed in a high-risk population, it does have some value in identifying pregnancies that may warrant more frequent blood pressure assessment.

The umbilical artery (UA) was the first vessel to be studied by Doppler ultrasonography. By about 15 weeks of gestation, diastolic flow can be identified in the UA. With advancing gestational age, the end-diastolic velocity increases secondary to the decrease in placental resistance. This is reflected in decreases in the S/D or PI. As the chorionic vascular bed undergoes an atherosclerotic-like process, local ischaemia and necrosis results. The Umbilical Artery shows increasing impedance that initially blunts forward flow during diastole, and ultimately reverses it at a later stage. These findings have been associated with adverse perinatal outcome. Once reversal of diastolic flow is identified, administration of steroids for fetal lung maturity in the premature fetus and delivery must be considered.

Middle cerebral artery (MCA) is another vessel well characterized by Doppler and has been shown to be affected by IUGR as well. MCA normally exhibits low amplitude of diastolic flow which increases in the presence of fetal hypoxia as a marker of cerebral vasodilation. This most commonly represents a later stage in the hypoxic process and typically occurs after changes in the uterine artery.
Dhand et al. [5] compared MCA Doppler indices with umbilical artery Doppler indices in a prospective study of 121 women of which 71 were high risk women with growth restricted fetuses and 50 women had healthy fetuses. The predictive value of Doppler PI for detecting abnormal fetal outcome was $94 \%$ in MCA as against $83 \%$ for umbilical artery. The sensitivity was $71 \%$ for MCA versus $44 \%$ for umbilical artery. Thus, the authors concluded that MCA Doppler indices were a better predictor for fetal outcome in IUGR when compared with umbilical artery in terms of sensitivity and predictive value.

The sequence of abnormal events that herald adverse perinatal outcome begins with an absence of UA end diastolic flow. Later findings include abnormal Doppler pulsatility of the MCA (with decreased PI) and abnormal ductus venosus flow (absent or reversed flow during atrial contraction) and reversed flow in the UA. These changes are significantly associated with perinatal death.

Management of the fetus with abnormal Doppler indices is gestational age dependant. In the mature fetus, there is little to be gained by continuing pregnancy and the time course to decompensation is generally shorter in these fetuses, therefore delivery is recommended. Induction of labour would be reasonable in those with re-assuring fetal heart tracing. In the immature fetus close monitoring is necessary.

Once absent end-diastolic flow in the UA is demonstrated, the biophysical profile (BPP) and Doppler indices should be done twice weekly and daily fetal kick counts is recommended. Once reversal of flow in the UA or cephalisation in the MCA is present, hospitalization with continuous oxygen therapy, bed rest, daily BPP and daily Doppler are indicated. Steroids for fetal lung maturity should be administered.

A pulsatile pattern in the DV Doppler is highly suggestive of fetal acidemia and is an indication for delivery. A fetus with either reversed end diastolic flow in the UA and/or a pulsatile DV pattern has little reserve and will not tolerate labour most likely.

3-D power Doppler is particularly sensitive for detecting low velocity flow which is then rendered to give a detailed image of fine vascular structures. Since tertiary stem villi can be visualized, there is a potential for earlier detection of abnormal placentation to detect pregnancies at high risk for pre-eclampsia or IUGR, or evaluation of a suspected placenta abruption or accreta [6].

\section{Placental MRI in Intrauterine Growth Restriction}

Fetal MRI is now established as an adjunct to ultrasonography in the diagnosis of fetal abnormalities. Damodaran et al. [7] studied MR imaging of the placenta in singleton growth restricted fetuses. The authors found that there was 
a significant increase in the placental volume affected by pathology in growth restricted fetuses. The placental appearance was also thickened and globular, with an increase in the placental thickness to volume ratio. Although placental volume increased with increasing gestation, it remained reduced in the growth restricted fetuses. The authors concluded that MRI appearance of the placenta provides an indication of the severity of the underlying disease process in fetal growth restriction.

\section{Treatment of Fetal Growth Restriction due to Placental Insufficiency}

Bed rest in hospital or at home is widely advised. This allows for close monitoring. However the benefits of bed rest must be balanced with the risk of thrombosis. Gulmezoglu and Hofmeyr evaluated the role of bed rest for impaired fetal growth. The authors compared bed rest with ambulatory management for women with impaired fetal growth. There were differences in the fetal weights and birth weights in both the groups but the difference was not statistically significant [8].

\section{Maternal Dietary Supplementation}

Maternal dietary supplementation through balanced caloric intake rather than specific protein supplementation has a variable effect on fetal growth. The effect is small, though fetal weights have been shown to increase by $100-300 \mathrm{~g}$. Ramakrishnan et al. [9] conducted a randomized double blind placebo controlled study on the effect of docosahexaenoic acid supplementation during pregnancy on gestational age and size at birth. The authors concluded that prenatal DHA supplementation of primigravid women may result in increased birth size in a population where DHA intakes are low.

\section{Nitric Oxide Donors}

L-Arginine improves Uteroplacental blood flow to overcome placental ischemia by increasing Nitric oxide. This results in vasodilation of uterine arteries. Neri et al. [10] evaluated the effects of L-arginine (ARG) infusion, the nitric oxide substrate on the uteroplacental circulation in the third trimester. Three groups of nine women each were infused with $30 \mathrm{~g}$ ARG for $30 \mathrm{~min}$. One group served as a control. The remaining two groups had IUGR, one with increased resistance in uteroplacental circulation and one without increased resistance. The authors found no haemodynamic changes in the utero-umbilical circulation. They found that serum nitrites/nitrates as well as serum growth hormone levels were significantly raised by ARG.
The authors also reported a significant decrease in resistance in the women where the IUGR was due to elevated resistance. They concluded that ARG infusion affects uteroplacental circulation in women with IUGR due to elevated resistance. Such an action is specific and appears to be mediated by a release of nitric oxide. Rytlewski et al. [11] studied the influence of oral supplementation with low dose of ARG on biophysical profile, feto-placental circulation and neonatal outcome in preeclampsia. This was a randomized, placebo-controlled, double-blind, clinical trial. Oral therapy with $3 \mathrm{~g}$ of ARG daily or placebo was given as a supplement to standard therapy. The results showed that $\mathrm{L}-$ arginine treatment accelerated fetal weight gain and improved biophysical profile. Starting from the 3rd week of therapy, the umbilical artery pulsatility indices values were significantly lower in the ARG group. Neonates in this group revealed higher Apgar scores. The authors concluded that supplementary treatment with oral ARG seems to be promising in improving foetal well-being and neonatal outcome as well as in prolonging pregnancy complicated with pre-eclampsia.

\section{Low Dose Aspirin}

The use of low-dose aspirin for the prevention and treatment of pre-eclampsia and intrauterine growth restriction has been studied extensively. Leitich et al. [12] performed a meta analysis of low dose aspirin for the prevention of IUGR. The use of aspirin showed a significant reduction in IUGR and a nonsignificant reduction in perinatal mortality. Subgroup analyses revealed that aspirin was effective at lower doses between 50 and $80 \mathrm{mg} / \mathrm{day}$, but that the preventive effect was greater at higher doses between 100 and $150 \mathrm{mg} /$ day and among women entered before the $17 \mathrm{th}$ week of gestation. The authors concluded that low dose aspirin should not be used routinely in pregnant women. The indications for the use of low dose aspirin would include preexisting chronic hypertension, a history of recurring pre-eclampsia and hypertension prior to 20 weeks and associated autoimmune disorders such as SLE, a positive anticardiolipin antibody test and the presence of lupus anticoagulant. A Multicentric study FLASP (FOGSI Low Dose Aspirin Study) was carried out by FOGSI to assess the effectiveness of low dose aspirin for prevention \& treatment of pre-eclampsia \& IUGR. The incidence of IUGR \& pre-eclampsia were significantly reduced in patients who received prophylactic low dose aspirin prior to 16 weeks of gestation. One of the largest collaborative studies was CLASP-Collaborative Low Dose Aspirin studies in pregnancy. This study recommended the use of Low Dose aspirin in women to be specially liable to early onset of pre-eclampsia severe enough to need preterm delivery. 
Heparin

Heparin plays an important role in preventing complications of the APA syndrome. Heparin prevents pregnancy loss by inhibiting complement activation on trophoblast in addition to its anticoagulant effect. Unfractionated heparin or low molecular weight heparin can be used. The main danger of heparin therapy in pregnancy is osteoporosis because its use in pregnancy is prolonged and pregnancy and lactation also cause reversible bone demineralization. Adequate calcium and Vit D3 intake and moderate exercise is necessary to prevent vertebral collapse. Bone density improves once heparin is discontinued.

Low molecular weight heparins (LMWH) have fewer complications than conventional heparin and are being more frequently used in pregnancy safely. LMWH inhibits factor $\mathrm{Xa}$ and has an antithrombotic effect while heparin has, in addition, an anticoagulant effect through its action on antithrombin III and factor IIa. Thus, bleeding complications are few with little alteration of PT and APTT. It can be given as once daily and has a lower risk of thrombocytopenia and osteoporosis. Both heparin and LMWH do not cross the placenta and fetal complications have not been reported. Enoxaparin $40 \mathrm{mg}$ per day subcutaneously or Dalteparin 5000 units per day is injected from the time of confirmation of pregnancy until delivery.

\section{Sildenafil Citrate}

Sildenafil citrate, a specific phosphodiesterase inhibitor, is increasingly used for pulmonary hypertension in pregnancy. Sildenafil is also emerging as a potential candidate for the treatment of intra-uterine growth retardation and for premature labour. Maharaj et al. [13] studied the effects and mechanisms of action of sildenafil citrate in human chorionic arteries ex vivo. In a series of pharmacologic studies, the effects of sildenafil citrate in pre-constricted chorionic plate arterial rings were determined. Their results showed that phosphodiesterase-5-mRNA and protein was demonstrated in human chorionic plate arteries. Sildenafil produced dose dependant vasodilatation. The authors concluded that sildenafil citrate vasodilated the feto-placental circulation via a cGMP dependant mechanism involving increased responsiveness to nitrous oxide. Von Dadelszen et al. [14] studied the role of sildenafil citrate therapy for severe early onset intrauterine growth restriction. Women were offered sildenafil citrate $25 \mathrm{mg}$ three times daily until delivery if their pregnancy was complicated by early onset IUGR (AC $<5$ th centile) and either the gestational age was less than 25 weeks or the fetal weight was $<600 \mathrm{~g}$. The authors found that sildenafil growth was associated with increased AC growth (odds ratio 12.9) Randomised controlled trial data are required to determine whether sildenafil improves perinatal outcome for early onset IUGR patients.

\section{Surveillance of the Growth-Retarded Fetus}

\section{Fetal Kick Count}

A reduction in fetal movements is a cause for concern and anxiety. Fetal movements follow a circadian pattern and are expressions of fetal well being [15]. Decreased fetal movements are regarded as a marker for suboptimal intrauterine conditions. The fetus responds to chronic hypoxia by conserving energy and the subsequent reduction of fetal movements is an adaptive mechanism to reduce oxygen consumption.

\section{Fetal Heart Rate Monitoring}

Fetal heart rate monitoring will show a sequence of changes that correlate with worsening in the fetal situation. The usual order is lack of accelerations, decreased variability, and onset of spontaneous decelerations. These changes are dependant on the severity of fetal compromise and the gestational age of the fetus. At less than 32 weeks it is unusual to obtain an accelerative pattern even if the fetus is not compromised.

FHR monitoring is a sensitive indicator of fetal hypoxia and acidosis but lacks specificity and has a significant number of false positive results.

\section{Biophysical Profile}

The BPP is a combination of the observation of the fetal behavior with ultrasound (fetal breathing movements, fetal movements, fetal tone and amniotic fluid volume) and FHR monitoring and is a sensitive test to determine exhaustion of fetal reserve.

\section{Delivery of the Growth Restricted Fetus}

The optimal management strategy is to avoid delivery of the preterm infant that is adequately compensating for the placental dysfunction and to recommend delivery when initial signs of acidemia are detected. The following changes are suggestive of beginning of fetal acidemia.

- Fetal Heart rate monitoring: No accelerations, absent or minimal variability

- Umbilical artery Doppler: Absent diastolic flow

- Bio Physical Profile 6 
- Ductus venosus: Decreased or absent forward flow during atrial contraction

The full-term fetus has a large capacity to tolerate the hypoxic stress of labour. This capacity is substantially reduced in fetal growth restriction due to the marked depletion of energy stores in the liver and subcutaneous tissues. With hypoxia, the energy reserves are rapidly consumed and the fetus must switch to anerobic metabolism for the generation of energy. Unfortunately, anaerobic metabolism produces a large number of hydrogen ions, and metabolic acidosis appears. Thus, intrapartum asphyxia is the major cause of perinatal morbidity and mortality in fetal growth restriction. Therefore, when umbilical Doppler shows absent or reversed diastolic flow, delivery by caesarean section is indicated. Vaginal delivery may be attempted with close monitoring in patients with increased resistance in the umbilical arteries but Caesarean delivery should be anticipated in a large number of them.

Fetal heart rate should be carefully monitored during labour and alterations of the fetal heart rate suggesting fetal compromise should be followed by caesarean delivery. The second stage of labour requires special attention. In most cases it is preferable to avoid pushing during the second stage and let the fetus descend under the exclusive effect of uterine contractions. It is not recommended to prolong the duration of the second stage for more than $2 \mathrm{~h}$ in nulliparous and $1 \mathrm{~h}$ in multiparous patients [16].

\section{Intrapartum Monitoring}

\section{Fetal Heart Rate Auscultation}

Intrapartum surveillance is mainly focused upon detecting fetal asphyxia in order to prevent perinatal death or future neurodevelopmental handicap. Bradycardia, tachycardia and irregular heart rate are signs of asphyxia.

\section{Electronic Fetal Monitoring}

Uterine contractions of labour reduce the uteroplacental blood flow and/or compress the umbilical cord depending on its position and the quantity of amniotic fluid. Reduction of blood flow may compromise fetuses which have preexisting hypoxia. The features of reduced baseline variability, decelerations, absence of accelerations suggest hypoxia in the fetus.

\section{Fetal Pulse Oximetry}

Fetal pulse oximetry appears to be a promising new tool in intrapartum fetal surveillance. Not only is it accurate and rapid in its measurement of fetal oxygenation, it also affords direct assessment (rather than indirect assessment, as with FHR monitoring) of fetal oxygenation and peripheral tissue perfusion. Fetal pulse oximeters measure the fraction of light that is unabsorbed after being passed through a pulsatile vascular bed. Sensors are placed on a skin surface such as the fetal head or face. Sensor placement is not difficult at cervical dilatations of more than $2 \mathrm{~cm}$ [17].

\section{Scalp Blood pH}

In 1962, Saling introduced fetal scalp blood sampling during labour. This is a useful aid to determine fetal acidosis when difficulties arise with interpretation of abnormal CTG tracings. With $\mathrm{CO}_{2}$ accumulation, due to reduced placental gas exchange, $\mathrm{pH}$ decreases as a result of respiratory acidosis. Increasing hypoxia leads to anaerobic metabolism resulting in lactate and hydrogen ion $(\mathrm{H}+)$ production. Low $\mathrm{pH}$ has been used as one of the best available measures for identification of asphyxia during labour and delivery. Although used effectively in many centres abroad, very few use this method in India.

\section{Conclusions}

The main antepartum complications of IUGR fetuses are an increased incidence of oligohydramnios, antepartum fetal distress and stillbirth. Intrapartum complications are fetal hypoxia, acidosis and high rate of caesarean delivery. Neonatal complications include hypoglycemia, hyperbilirubinemia, meconium aspiration, persistent fetal circulation, hypoxic-ischaemic encephalopathy, hypocalcemia, hyperviscosity syndrome and necrotizing enterocolitis. Doppler assessment of the uterine, umbilical, middle cerebral vessels is used to identify placental insufficiency.

The most important tests to follow the fetus with placental insufficiency are the fetal heart rate monitoring and umbilical and middle cerebral artery Doppler. As long as the fetal monitoring is normal and Doppler does not show fetal decompensation (absent end diastolic flow or reversed flow) expectant management in the preterm fetus is adequate.

\section{References}

1. Karowicz-Bilinska A, Kedziora-Kornatowska K, Bartosz G. Indices of oxidative stress in pregnancy with fetal growth restriction. Informa Health Care. 2007;41:870-3.

2. France J. Biochemical prediction of the low birth weight growth restricted baby. In: Tambyraja and Mongelli, editors. The low 
birth weight baby. Obstetrics and gynecology in perspective. Hyderabad: Orient Longman Private Limited; 2003.

3. Otiv S, Coyaji K. Fetal growth restriction. In: Krishna U, Shah D, Salvi V, Sheriar N, Damania K, editors. Pregnancy at risk. 5th ed. New Delhi: Jaypee Brothers Medical Publishers (P) Ltd.; 2010.

4. Zimmermann P, Eirio V, Koskinen J, et al. Doppler assessment of the uterine and uteroplacental circulation in the second trimester in pregnancies at high risk for pre-eclampsia and or intrauterine growth retardation: comparison between different Doppler parameters. Ultrasound Obstet Gynecol. 2001;18:441-9.

5. Dhand H, Kumar KH, Dave A. Middle cerebral artery Doppler indices better predictor for fetal outcome in IUGR. J Obstet Gynecol India. 2011;61:166-71.

6. O`Neill AM, Burd ID, Sabogal JC, et al. Doppler ultrasound in obstetrics: current advances. In: Studd J, editors. Progress in obstetrics and gynaecology. 17th ed. New Delhi: Elsevier; 2007.

7. Damodaram M, Story L, Eixarch E, et al. Placental MRI in fetal growth restriction. Placenta. 2010;31(6):491-8.

8. Gülmezoglu AM, Hofmeyr GJ. Bed rest in hospital for suspected impaired fetal growth. Cochrane Database Syst Rev. 2000; 2:CD000034.

9. Ramakrishnan U, Stein AD, Parra-Cabrera S, Wang M, ImhoffKunsch B, Juárez-Márquez S, Rivera J, Martorell R. Effects of docosahexaenoic acid supplementation during pregnancy on gestational age and size at birth: randomized, double-blind, placebo-controlled trial in Mexico. Food Nutr Bull. 2010;31(2 Suppl):S108-16.
10. Neri I, Mazza V, Galassi MC, et al. Effects of L-arginine on utero-placental circulation in growth related fetuses. Acta Obstet et Gynecol Scand. 1996; 75:208-212.

11. Rytlewski K, Olszanecki R, Lauterbach R, et al. Effects of oral $\mathrm{L}$-arginine on the foetal condition and neonatal outcome in preeclampsia: a preliminary report. Basic Clin Pharmacol Toxicol. 2006; 99(2):146-152.

12. Leitich H, Egarter C, Husslein P, et al. A meta-analysis of low dose aspirin for the prevention of intrauterine growth retardation. Br J Obstet Gynaecol. 1997;104(4):450-9.

13. Maharaj CH, O`Toole D, Lynch T, et al. Effects and mechanisms of action of sildenafil citrate in human chorionic arteries. Reprod Biol Endocrinol. 2009;7:34.

14. Von Dadelszen P, Dwinnell S, Magee LA, et al. Sildenafil citrate therapy for severe early-onset intrauterine growth restriction. BJOG. 2011;118(5):624-8.

15. Jassawalla MJ. Reduced fetal movements: interpretation and action. J Obstet Gynecol India. 2011;61(2):141-3.

16. Daftary SN, Bhide AG. Fetal growth restriction. In: Fernando A, Daftary SN, Bhide AG, editors. Practical guide to high-risk pregnancy and delivery-a South Asian perspective. Noida: Elsevier; 2008.

17. Yam J, Chua S, Arulkumaran S. Fetal pulse oximetry. In: Arulkumaran S, Jenkins HML, editors. Perinatal asphyxia. Hyderabad: Orient Longman Limited; 2000. 